\title{
Uncertainty principles for the Fourier and the short-time Fourier transforms
}

Cite as: J. Math. Phys. 62, 113501 (2021); https://doi.org/10.1063/5.0047191

Submitted: 11 February 2021 • Accepted: 11 October 2021 • Published Online: 01 November 2021

(iD) Anirudha Poria

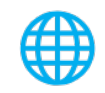

View Online

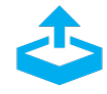

Export Citation

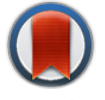

\section{ARTICLES YOU MAY BE INTERESTED IN}

Angular-radial integrability of Coulomb-like potentials in Dirac equations

Journal of Mathematical Physics 62, 113505 (2021); https://doi.org/10.1063/5.0055250

The Robin problem on rectangles

Journal of Mathematical Physics 62, 113503 (2021); https://doi.org/10.1063/5.0061763

Generalized Casimir operators for Lie superalgebras

Journal of Mathematical Physics 62, 101703 (2021); https://doi.org/10.1063/5.0056538

\section{Sign up for Alerts}

Journal of

Mathematical Physics

Receive the latest research updates 


\title{
Uncertainty principles for the Fourier and the short-time Fourier transforms
}

\author{
Cite as: J. Math. Phys. 62, 113501 (2021); doi: 10.1063/5.0047191 \\ Submitted: 11 February 2021 - Accepted: 11 October 2021 • \\ Published Online: 1 November 2021
}

\section{Anirudha Poria}

\section{AFFILIATIONS}

Department of Mathematics, Indian Institute of Science, Bengaluru 560012, Karnataka, India

a) Author to whom correspondence should be addressed: anirudhamath@gmail.com

\section{ABSTRACT}

The aim of this paper is to establish a few uncertainty principles for the Fourier and the short-time Fourier transforms. In addition, we discuss an analog of the Donoho-Stark uncertainty principle and provide some estimates for the size of the essential support of the short-time Fourier transform.

Published under an exclusive license by AIP Publishing. https://doi.org/10.1063/5.0047191

\section{INTRODUCTION AND STATEMENT OF THE RESULTS}

The uncertainty principle states that a non-zero function and its Fourier transform cannot be simultaneously sharply localized. We consider the Fourier transform on $\mathbb{R}^{d}$ to be normalized as

$$
\hat{f}(\xi)=\int_{\mathbb{R}^{d}} f(x) e^{-2 \pi i x \cdot \xi} d x
$$

where $x \cdot \xi$ is the standard inner product on $\mathbb{R}^{d}$. There are various forms of the uncertainty principle. The most remarkable result is due to Beurling, ${ }^{1}$ which states the following theorem:

Theorem 1.1 (Beurling). Let $f \in L^{2}\left(\mathbb{R}^{d}\right)$ be such that

$$
\iint_{\mathbb{R}^{2 d}}|f(x) \hat{f}(\xi)| e^{2 \pi|x \cdot \xi|} d x d \xi<\infty
$$

Then, $f=0$ almost everywhere.

Its proof was published much later in 1991 by Hörmander. ${ }^{1}$ We can obtain the well-known uncertainty principles of Hardy, Cowling-Price, and Gelfand-Shilov as corollaries to Theorem 1.1 (see Ref. 2). For the purpose of this paper, we state here Hardy's and Cowling-Price's theorems. In 1933, $\mathrm{Hardy}^{3}$ proved the following uncertainty principle:

Theorem 1.2 (Ref. 3). Let $f \in L^{2}\left(\mathbb{R}^{d}\right)$ and assume that

$$
|f(x)| \leq C e^{-a \pi x^{2}} \quad \text { and } \quad|\hat{f}(\xi)| \leq C e^{-b \pi \xi^{2}}
$$

for some constants $a, b, C>0$. Then, the following three cases can occur.

(i) If $a b=1$, then $f(x)=C e^{-a \pi x^{2}}$.

(ii) If $a b>1$, then $f \equiv 0$.

(iii) If $a b<1$, then any finite linear combination of Hermite functions satisfies these decay conditions. 
In 1983, Cowling and Price ${ }^{4}$ generalized this theorem by replacing point wise Gaussian bounds for $f$ by Gaussian bounds in $L^{p}$ sense and in $L^{q}$ sense for $\hat{f}$ as well. More precisely, they proved the following theorem:

Theorem 1.3 (Ref. 4). Let $f: \mathbb{R}^{d} \rightarrow \mathbb{C}$ be a measurable function such that

(i) $\left\|e^{a \pi x^{2}} f\right\|_{p}<\infty$,

(ii) $\left\|e^{b \pi \xi^{2}} \hat{f}\right\|_{q}<\infty$,

where $a, b>0$ and $1 \leq p, q \leq \infty$ such that $\min (p, q)$ is finite. If $a b \geq 1$, then $f=0$ almost everywhere. If ab $<1$, then there exist infinitely many linearly independent functions satisfying $(i)$ and $(i i)$.

Beurling's theorem was further generalized in 2003 by Bonami, Demange, and Jaming ${ }^{5}$ as follows:

Theorem 1.4. Let $f \in L^{2}\left(\mathbb{R}^{d}\right)$ be such that

$$
\iint_{\mathbb{R}^{2 d}} \frac{|f(x) \hat{f}(\xi)|}{(1+|x|+|\xi|)^{N}} e^{2 \pi|x \cdot \xi|} d x d \xi<\infty
$$

for some $N \geq 0$. Then, $f=0$ almost everywhere whenever $N \leq d$. If $N>d$, then $f(x)=P(x) e^{-a \pi x^{2}}$, where $P$ is a polynomial of degree $<\frac{N-d}{2}$ and $a>0$.

Over the years, analog of Beurling's theorem have been extended to different settings (see Ref. 2). For a more detailed study of uncertainty principles, we refer to the book of Havin and Jöricke. ${ }^{6}$ In time-frequency analysis, another tool of investigation is the short-time Fourier transform (STFT). We first write the definition of the STFT. given by

Let $g \in \mathcal{S}\left(\mathbb{R}^{d}\right)$ be a fixed window function. Then, the STFT of $f \in \mathcal{S}^{\prime}\left(\mathbb{R}^{d}\right)$ with respect to $g$ is defined to be the function on $\mathbb{R}^{d} \times \hat{\mathbb{R}}^{d}$

$$
V_{g} f(x, \xi)=\int_{\mathbb{R}^{d}} f(t) \overline{g(t-x)} e^{-2 \pi i \xi \cdot t} d t
$$

Gröchenig and Zimmermann ${ }^{7}$ showed that it is possible to derive new uncertainty principles for the STFT from uncertainty principles for the pair $(f, \hat{f})$ using a fundamental identity for the STFT. They proved a version of Hardy's theorem for the STFT.

Theorem 1.5. Let $(g, f) \in \mathcal{S} \times \mathcal{S}^{\prime}\left(\mathbb{R}^{d}\right)$, and assume that $\left|V_{g} f(x, \xi)\right| \leq C e^{-\pi\left(x^{2}+\xi^{2}\right) / 2}$ and that $V_{g} f$ does not vanish identically. Then, $V_{g} f(x, \xi)=C e^{2 \pi i\left(\zeta_{0} \cdot x-\xi \cdot z_{0}\right)} e^{-\pi\left(x^{2}+\xi^{2}\right) / 2} e^{-\pi i \xi \cdot x}$ for some $\left(z_{0}, \zeta_{0}\right) \in \mathbb{R}^{d} \times \hat{\mathbb{R}}^{d}$, and $f$ and $g$ are multiples of $e^{2 \pi i \zeta_{0} \cdot t} e^{-\pi\left(t-z_{0}\right)^{2}}$.

Considerable attention has been paid to prove an analog of Beurling's theorem for the STFT (see Refs. 5 and 8-10). Bonami, Demange, and Jaming ${ }^{5}$ proved the following version of Beurling's theorem for the STFT. They used the $L^{2}$-norm instead of the $L^{1}$-norm of the STFT.

Theorem 1.6. Let $f, g \in L^{2}\left(\mathbb{R}^{d}\right)$ be non-identically vanishing. If

$$
\iint_{\mathbb{R}^{2 d}} \frac{\left|V_{g} f(x, \xi)\right|^{2}}{(1+|x|+|\xi|)^{N}} e^{\pi\left(x^{2}+\xi^{2}\right)} d x d \xi<\infty,
$$

then there exists $a, w \in \mathbb{R}^{d}$ such that both $f$ and $g$ are of the form $P(x) e^{2 \pi i w \cdot x} e^{-\pi(x-a)^{2}}$, where $P$ is a polynomial.

Demange $^{8}$ improved the above theorem to the following sharper version of Beurling's theorem for the STFT:

Theorem 1.7. Let $f, g \in L^{2}\left(\mathbb{R}^{d}\right)$. If there exists an $N \geq 0$ such that

$$
\iint_{\mathbb{R}^{2 d}} \frac{\left|V_{g} f(x, \xi)\right|}{(1+|x|+|\xi|)^{N}} e^{\pi|x \cdot \xi|} d x d \xi<\infty
$$

then either $f$ or $g$ is identically zero or both can be written as

$$
f(x)=P(x) e^{-a x^{2}-2 \pi i w \cdot x} \text { and } g(x)=Q(x) e^{-a x^{2}-2 \pi i w \cdot x},
$$

with $P$ and $Q$ polynomials whose degrees satisfy $\operatorname{deg}(P)+\operatorname{deg}(Q)<N-d, w \in \mathbb{C}^{d}$ and $a>0$. The converse is also true. In particular, for $N \leq d$, $f$ or $g$ is identically vanishing. 
It has been conjectured that a result similar to Beurling's theorem is also true for the STFT. Gröchenig ${ }^{10}$ posed the following conjecture as a version of Beurling's theorem for the STFT:

Conjecture 1.8. Assume that $f, g \in L^{2}\left(\mathbb{R}^{d}\right)$. If

$$
\iint_{\mathbb{R}^{2 d}}\left|V_{g} f(x, \xi)\right| e^{\pi|x \cdot \xi|} d x d \xi<\infty
$$

then $f \equiv 0$ or $g \equiv 0$.

However, so far, up to our knowledge, this result has not been proved. However, Gröchenig ${ }^{10}$ proved a weaker version of this conjecture as follows:

Theorem 1.9. Assume that $f, g \in L^{2}\left(\mathbb{R}^{d}\right)$. If

$$
\iint_{\mathbb{R}^{2 d}}\left|V_{g} f(x, \xi)\right| e^{\pi\left(x^{2}+\xi^{2}\right) / 2} d x d \xi<\infty,
$$

then $f \equiv 0$ or $g \equiv 0$.

Furthermore, Gröchenig obtained an estimate for the size of the essential support of $V_{g} f$, analogous to the uncertainty principle of Donoho and $\operatorname{Stark}^{11}$ for the pair $(f, \hat{f})$. Several interesting versions of uncertainty principle have been studied by various authors for the STFT. We refer the reader to Refs. $12-14$ and the references therein.

The aim of this paper is to prove Conjecture 1.8 and a few uncertainty principles for the Fourier and the STFT. We investigate the following problems:

If $\left\|e^{\pi\left(x^{2}+\xi^{2}\right) / 2} V_{g} f\right\|_{L^{p}\left(\mathbb{R}^{2 d}\right)}<\infty,\left\|e^{\pi|x \cdot \xi|} V_{g} f\right\|_{L^{p}\left(\mathbb{R}^{2 d}\right)}<\infty$, or $\left\|e^{2 \pi|x \cdot \xi|} f \hat{f}\right\|_{L^{p}\left(\mathbb{R}^{2 d}\right)}<\infty$, then what we can say about the functions $f$ and $g$. More precisely, we establish the following problems:

Theorem 1.10. Let $1 \leq p<\infty$ and $f, g \in L^{2}\left(\mathbb{R}^{d}\right)$ be non-identically vanishing. If

$$
\iint_{\mathbb{R}^{2 d}}\left|V_{g} f(x, \xi)\right|^{p} e^{\pi p\left(x^{2}+\xi^{2}\right) / 2} d x d \xi<\infty
$$

then $f \equiv 0$ or $g \equiv 0$.

Theorem 1.11. Let $1 \leq p<\infty$ and $f, g \in L^{2}\left(\mathbb{R}^{d}\right)$ be non-identically vanishing. If

$$
\iint_{\mathbb{R}^{2 d}}\left|V_{g} f(x, \xi)\right|^{p} e^{\pi p|x \cdot \xi|} d x d \xi<\infty,
$$

then $f \equiv 0$ or $g \equiv 0$.

Theorem 1.12. Let $1 \leq p<\infty$ and $f \in L^{2}\left(\mathbb{R}^{d}\right)$ be such that

$$
\iint_{\mathbb{R}^{2 d}}|f(x) \hat{f}(\xi)|^{p} e^{2 \pi p|x \cdot \xi|} d x d \xi<\infty
$$

Then, $f \equiv 0$.

Finally, we extend the results of Gröchenig ${ }^{10}$ and prove the following versions of the uncertainty principle about an estimate on the size of the essential support of the STFT:

Theorem 1.13. Let $f$ and $g \in L^{2}\left(\mathbb{R}^{d}\right)$. If $U \subseteq \mathbb{R}^{2 d}$ and $\epsilon \geq 0$ are such that

$$
\iint_{U}\left|V_{g} f(x, \xi)\right| d x d \xi \geq(1-\epsilon)\|f\|_{2}\|g\|_{2}
$$

then

$$
|U| \geq(1-\epsilon)^{\frac{p}{p-1}}\left(\frac{p}{2}\right)^{\frac{d}{p-1}} \quad \text { for all } p \geq 2
$$


Theorem 1.14. Suppose that $f, g \in L^{2}\left(\mathbb{R}^{d}\right), U \subseteq \mathbb{R}^{2 d}$, and $\epsilon \geq 0$ are such that

$$
\iint_{U}\left|V_{g} f(x, \xi)\right|^{p} d x d \xi \geq(1-\epsilon)\left\|V_{g} f\right\|_{1}^{p}
$$

Then,

$$
|U| \geq 2^{\frac{2 p d}{2-p}}(1-\epsilon)^{\frac{2}{2-p}} \quad \text { for all } 1 \leq p<2 .
$$

This paper is organized as follows: In Sec. II, we recall some of the properties of the STFT. Then, in Sec. III, we prove the main results and discuss some consequences.

\section{THE SHORT-TIME FOURIER TRANSFORM}

Translation and modulation are defined by $T_{x} f(t)=f(t-x)$ and $M_{\xi} f(t)=e^{2 \pi i t \cdot \xi} f(t)$, where $t, x, \xi \in \mathbb{R}^{d}$. Using this notation, the STFT can be written as

$$
V_{g} f(x, \xi)=\left\langle f, M_{\xi} T_{x} g\right\rangle=\left(\overline{f \cdot T_{x} \bar{g}}\right)(\xi)
$$

For a detailed discussion of STFT, see Ref. 9. To prove uncertainty principles for the STFT, we need to construct an expression derived from $V_{g} f$ that is invariant under the $2 d$-dimensional Fourier transform. This kind of function was obtained by Jaming in Ref. 12 , which played a central role in obtaining certain uncertainty theorems for the STFT. We recall the following identities for the STFT from Ref. 10, which we need for the Proof of Theorem 1.10.

Lemma 2.1. Assume that $f_{1}, f_{2}, g_{1}, g_{2} \in L^{2}\left(\mathbb{R}^{d}\right)$. Then,

$$
\left(V_{g_{1}} \overline{f_{1} \overline{V_{g_{2}} f_{2}}}\right)(x, \xi)=\left(V_{f_{2}} f_{1} \overline{V_{g_{2}} g_{1}}\right)(-\xi, x) .
$$

Putting $f_{1}=f_{2}, g_{1}=g_{2}$, and $x=\xi=0$ in (2.1), we obtain the isometry property of the STFT,

$$
\left\|V_{g} f\right\|_{L^{2}\left(\mathbb{R}^{2 d}\right)}^{2}=\|f\|_{2}^{2}\|g\|_{2}^{2}
$$

Lemma 2.2. (i) For $f, g \in L^{2}\left(\mathbb{R}^{d}\right)$, the function

$$
F(x, \xi)=e^{2 \pi i x \cdot \xi} V_{g} f(x, \xi) V_{g} f(-x,-\xi)
$$

satisfies

$$
\hat{F}(x, \xi)=F(-\xi, x)
$$

(ii) Consider the family of functions defined as

$$
F_{(z, \zeta)}(x, \xi)=e^{2 \pi i x \cdot \xi} V_{g}\left(M_{\zeta} T_{z} f\right)(x, \xi) V_{g}\left(M_{\zeta} T_{z} f\right)(-x,-\xi) .
$$

Then,

$$
\widehat{F_{(z, \zeta)}}(x, \xi)=F_{(z, \zeta)}(-\xi, x) \quad \text { for all }(z, \zeta) \in \mathbb{R}^{2 d} .
$$

The above lemmas contain a fundamental identity for the STFT. They have been derived and used to prove certain uncertainty principles for the STFT (see Refs. 7, 10, and 12). The advantage of the identity is that the auxiliary function $F_{(z, \zeta)}$ inherits many properties from $V_{g} f$. For instance, if $V_{g} f$ possesses a certain decay, then $F_{(z, \zeta)}$ has a similar decay.

\section{PROOFS OF MAIN RESULTS}

A simple consequence of the Cowling-Price's theorem is obtained in the following lemma:

Lemma 3.1. Let $1 \leq p<\infty$ and $f \in L^{2}\left(\mathbb{R}^{d}\right)$ be such that

$$
\iint_{\mathbb{R}^{2 d}}|f(x) \hat{f}(\xi)|^{p} e^{\pi p\left(x^{2}+\xi^{2}\right)} d x d \xi<\infty .
$$


Then, $f \equiv 0$.

Proof. Let $e_{\pi}(x)=e^{\pi x^{2}}$ for $x \in \mathbb{R}^{d}$. If $f \in L^{2}\left(\mathbb{R}^{d}\right)$ satisfies (3.1), then we have

$$
\left\|e_{\pi} f\right\|_{p}^{p}\left\|e_{\pi} \hat{f}\right\|_{p}^{p}=\iint_{\mathbb{R}^{2 d}}|f(x) \hat{f}(\xi)|^{p} e^{\pi p\left(x^{2}+\xi^{2}\right)} d x d \xi<\infty .
$$

Thus, assumption (3.1) implies that $e_{\pi} f$ and $e_{\pi} \hat{f}$ are both in $L^{p}\left(\mathbb{R}^{d}\right)$ for $1 \leq p<\infty$. Hence, the conditions of Cowling-Price's theorem (Theorem 1.3) are satisfied for $f$, and we conclude that $f \equiv 0$.

Using a similar technique as in Theorem 2.7.2 (Ref. 10) and Lemma 3.1, we obtain the Proof of Theorem 1.10 as follows:

Proof of Theorem 1.10. Let $X=(x, \xi)$ and $Z=(z, \zeta) \in \mathbb{R}^{2 d}$. Let $U X=(-\xi, x)$ denote the rotation and $X^{2}=x^{2}+\xi^{2}$. We consider the family of functions defined in Lemma 2.2 as

$$
F_{(z, \zeta)}(x, \xi)=F_{Z}(X)=e^{2 \pi i x \cdot \xi} V_{g}\left(M_{\zeta} T_{z} f\right)(x, \xi) V_{g}\left(M_{\zeta} T_{z} f\right)(-x,-\xi) .
$$

Using Lemma $2.2(i i)$, we get $\widehat{F}_{Z}(\Omega)=F_{Z}(U \Omega)$. To apply Lemma 3.1, we have to prove that

$$
\int_{\mathbb{R}^{2 d}} \int_{\mathbb{R}^{2 d}}\left|F_{Z}(X) \widehat{F_{Z}}(\Omega)\right|^{p} e^{\pi p\left(X^{2}+\Omega^{2}\right)} d X d \Omega<\infty .
$$

Thus, it is enough to prove that

$$
\begin{aligned}
& \int_{\mathbb{R}^{2 d}} \int_{\mathbb{R}^{2 d}}\left|F_{Z}(X)\right|^{p}\left|F_{Z}(U \Omega)\right|^{p} e^{\pi p\left(X^{2}+\Omega^{2}\right)} d X d \Omega \\
& =\left(\int_{\mathbb{R}^{2 d}}\left|F_{Z}(X)\right|^{p} e^{\pi p X^{2}} d X\right)^{2}:=B(Z)^{2}<\infty .
\end{aligned}
$$

Since $\left|V_{g}\left(M_{\zeta} T_{z} f\right)(x, \xi)\right|=\left|V_{g} f(x-z, \xi-\zeta)\right|=\left|V_{g} f(X-Z)\right|$ and $\frac{1}{2}(X-Z)^{2}+\frac{1}{2}(-X-Z)^{2}=X^{2}+Z^{2}$, we can write the expression for $B(Z)$ as

$$
B(Z)=e^{-\pi p Z^{2}} \int_{\mathbb{R}^{2 d}}\left|V_{g} f(X-Z)\right|^{p} e^{\pi p(X-Z)^{2} / 2}\left|V_{g} f(-X-Z)\right|^{p} e^{\pi p(-X-Z)^{2} / 2} d X .
$$

Let $\Psi(X)=\left|V_{g} f(X)\right|^{p} e^{\pi p X^{2} / 2}$, then assumption (1.3) implies that

$$
\int_{\mathbb{R}^{2 d}} \Psi(X) d X=\int_{\mathbb{R}^{d}} \int_{\mathbb{R}^{d}}\left|V_{g} f(x, \xi)\right|^{p} e^{\pi p\left(x^{2}+\xi^{2}\right) / 2} d x d \xi<\infty,
$$

and therefore, $\Psi \in L^{1}\left(\mathbb{R}^{2 d}\right)$. Moreover,

$$
\begin{aligned}
B(Z) & =e^{-\pi p Z^{2}} \int_{\mathbb{R}^{2 d}} \Psi(X-Z) \Psi(-X-Z) d X \\
& =e^{-\pi p Z^{2}} \int_{\mathbb{R}^{2 d}} \Psi(X) \Psi(-2 Z-X) d X \\
& =e^{-\pi p Z^{2}}(\Psi * \Psi)(-2 Z) .
\end{aligned}
$$

Since $\Psi \in L^{1}\left(\mathbb{R}^{2 d}\right)$, we have $\Psi * \Psi \in L^{1}\left(\mathbb{R}^{2 d}\right)$ and hence $\Psi * \Psi(-2 Z)<\infty$. Thus, $B(Z)<\infty$ for almost all $Z \in \mathbb{R}^{2 d}$. Thus, condition (3.1) of Lemma 3.1 is satisfied for $F_{Z}(X)$, and we conclude that

$$
\left|F_{Z}(X)\right|=\left|V_{g} f(X-Z) V_{g} f(-X-Z)\right|=0
$$

for almost all $Z \in \mathbb{R}^{2 d}$. Since $F_{Z}(X)$ is jointly continuous in $X$ and $Z,(3.4)$ is true for all $X, Z \in \mathbb{R}^{2 d}$. Therefore,

$$
\left|F_{Z}(0)\right|=\left|V_{g} f(-Z)\right|^{2}=0 \quad \text { for all } Z \in \mathbb{R}^{2 d} .
$$

This implies that either $f \equiv 0$ or $g \equiv 0$. 
Proof of Theorem 1.11. Let $1 \leq p, q<\infty$ with $1 / p+1 / q=1$. We choose $N>0$ such that

$$
\iint_{\mathbb{R}^{2 d}} \frac{1}{(1+|x|+|\xi|)^{N q}} d x d \xi<\infty
$$

Then, using Hölder's inequality, we get

$$
\begin{aligned}
& \iint_{\mathbb{R}^{2 d}} \frac{\left|V_{g} f(x, \xi)\right|}{(1+|x|+|\xi|)^{N}} e^{\pi|x \cdot \xi|} d x d \xi \\
& \leq\left(\iint_{\mathbb{R}^{2 d}}\left|V_{g} f(x, \xi)\right|^{p} e^{\pi p|x \cdot \xi|} d x d \xi\right)^{\frac{1}{p}}\left(\iint_{\mathbb{R}^{2 d}} \frac{1}{(1+|x|+|\xi|)^{N q}} d x d \xi\right)^{\frac{1}{q}} .
\end{aligned}
$$

Thus, assumptions (1.4) and (3.5) imply that

$$
\iint_{\mathbb{R}^{2 d}} \frac{\left|V_{g} f(x, \xi)\right|}{(1+|x|+|\xi|)^{N}} e^{\pi|x \cdot \xi|} d x d \xi<\infty
$$

Hence, condition (1.2) of Theorem 1.7 is satisfied for $V_{g} f$, and we conclude that either $f$ or $g$ is identically zero or both can be written as

$$
f(x)=P(x) e^{-a x^{2}-2 \pi i w \cdot x} \text { and } g(x)=Q(x) e^{-a x^{2}-2 \pi i w \cdot x}
$$

with $P$ and $Q$ polynomials whose degrees satisfy $\operatorname{deg}(P)+\operatorname{deg}(Q)<N-d, w \in \mathbb{C}^{d}$ and $a>0$. Indeed, we show that if $f$ and $g$ are as in $(3.7)$ and $V_{g} f$ satisfies (1.4), then $f \equiv 0$ or $g \equiv 0$.

Let $f$ and $g$ are of the form given in (3.7), then

$$
V_{g} f(x, \xi)=R(x, \xi) e^{-\pi i x \cdot \xi} e^{-(\pi / 2) a^{-1}\left(\xi+2 i w_{2}\right)^{2}} e^{-(\pi / 2) a x^{2}} e^{-2 \pi i x \cdot w_{1}},
$$

where $R$ is a polynomial of $\operatorname{degree} \operatorname{deg}(P)+\operatorname{deg}(Q)$ and $w=w_{1}+i w_{2}$. Therefore,

$$
\left|V_{g} f(x, \xi)\right|=|R(x, \xi)| e^{-(\pi / 2) a^{-1}\left(\xi^{2}-4 w_{2}^{2}\right)} e^{-(\pi / 2) a x^{2}} .
$$

Since $V_{g} f$ satisfies (1.4), we have

$$
\begin{aligned}
& \iint_{\mathbb{R}^{2 d}}\left|V_{g} f(x, \xi)\right|^{p} e^{\pi p|x \cdot \xi|} d x d \xi \\
& =e^{2 \pi a^{-1} p w_{2}^{2}} \iint_{\mathbb{R}^{2 d}}|R(x, \xi)|^{p} e^{-(\pi / 2) p\left(a x^{2}+a^{-1} \xi^{2}-2|x||\xi|\right)} d x d \xi<\infty .
\end{aligned}
$$

It remains to show that this is only possible for $R \equiv 0$. We are linked to prove that

$$
\iint_{\mathbb{R}^{2 d}}|R(u, v)|^{p} e^{-|u-v|^{2}} d u d v=\infty
$$

for any non-zero polynomial $R$. However, non-vanishing polynomials are bounded below, say, for $|u|>A,|v|>A$, then

$$
\int_{|v|>A} \int_{|u|>A} e^{-|u-v|^{2}} d u d v=\infty
$$

This completes the proof.

Remark 3.2. Theorem 1.11 implies Theorem 1.10. To see this, assume that condition (1.3) of Theorem 1.10 is satisfied. Then,

$$
\iint_{\mathbb{R}^{2 d}}\left|V_{g} f(x, \xi)\right|^{p} e^{\pi p|x \cdot \xi|} d x d \xi \leq \iint_{\mathbb{R}^{2 d}}\left|V_{g} f(x, \xi)\right|^{p} e^{\pi p\left(x^{2}+\xi^{2}\right) / 2} d x d \xi<\infty .
$$

By Theorem 1.11, $f \equiv 0$ or $g \equiv 0$. 
Proof of Theorem 1.12. Let $1 \leq p, q<\infty$ with $1 / p+1 / q=1$. We choose $N>0$ such that

$$
\iint_{\mathbb{R}^{2 d}} \frac{1}{(1+|x|+|\xi|)^{N q}} d x d \xi<\infty
$$

Then, using Hölder's inequality, we get

$$
\begin{aligned}
& \iint_{\mathbb{R}^{2 d}} \frac{|f(x) \hat{f}(\xi)|}{(1+|x|+|\xi|)^{N}} e^{2 \pi|x \cdot \xi|} d x d \xi \\
& \leq\left(\iint_{\mathbb{R}^{2 d}}|f(x) \hat{f}(\xi)|^{p} e^{2 \pi p|x \cdot \xi|} d x d \xi\right)^{\frac{1}{p}}\left(\iint_{\mathbb{R}^{2 d}} \frac{1}{(1+|x|+|\xi|)^{N q}} d x d \xi\right)^{\frac{1}{q}} .
\end{aligned}
$$

Thus, assumptions (1.5) and (3.8) imply that

$$
\iint_{\mathbb{R}^{2 d}} \frac{|f(x) \hat{f}(\xi)|}{(1+|x|+|\xi|)^{N}} e^{2 \pi|x \cdot \xi|} d x d \xi<\infty .
$$

Hence, condition (1.1) of Theorem 1.4 is satisfied for $f$, and we conclude that $f=0$ almost everywhere whenever $N \leq d$ and if $N>d$, then $f(x)=P(x) e^{-a \pi x^{2}}$, where $P$ is a polynomial of degree $<\frac{N-d}{2}$ and $a>0$. Indeed, we show that if $f$ is of this form, then $f \equiv 0$.

Let $f(x)=P(x) e^{-a \pi x^{2}}$, then $\hat{f}(\xi)=Q(\xi) e^{-\pi a^{-1} \xi^{2}}$ for some polynomial Q. Since $f$ satisfies (1.5), we have

$$
\iint_{\mathbb{R}^{2 d}}|f(x) \hat{f}(\xi)|^{p} e^{2 \pi p|x \cdot \xi|} d x d \xi=\iint_{\mathbb{R}^{2 d}}|P(x)|^{p}|Q(\xi)|^{p} e^{-\pi p\left(a x^{2}+a^{-1} \xi^{2}-2|x||\xi|\right)} d x d \xi<\infty
$$

It remains to show that this is only possible for $P \equiv 0$. We are linked to prove that

$$
\iint_{\mathbb{R}^{2 d}}|P(u)|^{p}|Q(v)|^{p} e^{-|u-v|^{2}} d u d v=\infty
$$

for any non-zero polynomials $P$ and $Q$. However, non-vanishing polynomials are bounded below, say, for $|u|>A,|v|>A$, and then

$$
\int_{|v|>A} \int_{|u|>A} e^{-|u-v|^{2}} d u d v=\infty
$$

This completes the proof.

Remark 3.3. (i) If we consider $p=1$ in Theorems 1.10 and 1.11, then we obtain Theorem 1.9 and Conjecture 1.8, respectively.

(ii) If we consider $p=1$ in Theorem 1.12, then we obtain Theorem 1.1.

Next, we discuss an analog of the Donoho-Stark uncertainty principle and provide some estimates for the size of the essential support of $V_{g} f$. We start with the following lemma:

Lemma 3.4. Let $1 \leq p<\infty$ and $f, g \in L^{2}\left(\mathbb{R}^{d}\right)$. If $U \subseteq \mathbb{R}^{2 d}$ and $\epsilon \geq 0$ are such that

$$
\iint_{U}\left|V_{g} f(x, \xi)\right|^{p} d x d \xi \geq(1-\epsilon)\|f\|_{2}^{p}\|g\|_{2}^{p},
$$

then $|U| \geq 1-\epsilon$.

Proof. The Cauchy-Schwartz inequality implies that

$$
\left|V_{g} f(x, \xi)\right|=\left|\left\langle f, M_{\xi} T_{x} g\right\rangle\right| \leq\|f\|_{2}\|g\|_{2} \quad \text { for all }(x, \xi) \in \mathbb{R}^{2 d} .
$$

Therefore,

$$
(1-\epsilon)\|f\|_{2}^{p}\|g\|_{2}^{p} \leq \iint_{U}\left|V_{g} f(x, \xi)\right|^{p} d x d \xi \leq\left\|V_{g} f\right\|_{\infty}^{p}|U| \leq|U|\|f\|_{2}^{p}\|g\|_{2}^{p}
$$

and so $|U| \geq 1-\epsilon$.

Estimates obtained in Theorems 1.13 and 1.14 improve Lemma 3.4 and provide a stronger estimate on the size of the essential support of $V_{g} f$. To prove Theorems 1.13 and 1.14 , we use Lieb's ${ }^{13}$ uncertainty principle. 
Theorem 3.5 (Ref. 13). Assume that $f, g \in L^{2}\left(\mathbb{R}^{d}\right)$. Then,

$$
\iint_{\mathbb{R}^{2 d}}\left|V_{g} f(x, \xi)\right|^{p} d x d \xi \begin{cases}\leq\left(\frac{2}{p}\right)^{d}\left(\|f\|_{2}\|g\|_{2}\right)^{p} & \text { if } 2 \leq p<\infty, \\ \geq\left(\frac{2}{p}\right)^{d}\left(\|f\|_{2}\|g\|_{2}\right)^{p} & \text { if } 1 \leq p \leq 2 .\end{cases}
$$

Proof of Theorem 1.13. We first apply Hölder's inequality with exponents $q=p$ and $q^{\prime}=\frac{p}{p-1}$, and then in the second step, we use Lieb's inequality for $p \geq 2$, and we obtain

$$
\begin{aligned}
(1-\epsilon) & \|f\|_{2}\|g\|_{2} \leq \iint_{U}\left|V_{g} f(x, \xi)\right| d x d \xi \\
& \leq\left(\iint_{\mathbb{R}^{2 d}}\left|V_{g} f(x, \xi)\right|^{p} d x d \xi\right)^{\frac{1}{p}}\left(\iint_{\mathbb{R}^{2 d}} \chi_{U}(x, \xi)^{q^{\prime}} d x d \xi\right)^{\frac{p-1}{p}} \\
& \leq\left(\frac{2}{p}\right)^{\frac{d}{p}}\|f\|_{2}\|g\|_{2}|U|^{\frac{p-1}{p}} .
\end{aligned}
$$

Thus,

$$
|U| \geq(1-\epsilon)^{\frac{p}{p-1}}\left(\frac{p}{2}\right)^{\frac{d}{p-1}} \quad \text { for all } p \geq 2
$$

Proof of Theorem 1.14. Using Lieb's inequality for $p=1$ and (2.2), we obtain that

$$
(1-\epsilon)\left\|V_{g} f\right\|_{1}^{p} \geq(1-\epsilon) 2^{p d}\|f\|_{2}^{p}\|g\|_{2}^{p}=(1-\epsilon) 2^{p d}\left\|V_{g} f\right\|_{2}^{p} .
$$

On the other hand, using Hölder's inequality with exponents $q=\frac{2}{p}$ and $q^{\prime}=\frac{2}{2-p}$, for $1 \leq p<2$, we get

$$
\begin{aligned}
\iint_{U}\left|V_{g} f(x, \xi)\right|^{p} d x d \xi & \leq\left(\iint_{\mathbb{R}^{2 d}}\left|V_{g} f(x, \xi)\right|^{2} d x d \xi\right)^{\frac{p}{2}}\left(\iint_{\mathbb{R}^{2 d}} \chi_{U}(x, \xi)^{q^{\prime}} d x d \xi\right)^{\frac{2-p}{2}} \\
& =\left\|V_{g} f\right\|_{2}^{p}|U|^{\frac{2-p}{2}} .
\end{aligned}
$$

Combining these inequalities with (1.6), we obtain

$$
(1-\epsilon) 2^{p d}\left\|V_{g} f\right\|_{2}^{p} \leq|U|^{\frac{2-p}{2}}\left\|V_{g} f\right\|_{2}^{p}
$$

Thus,

$$
|U| \geq 2^{\frac{2 p d}{2-p}}(1-\epsilon)^{\frac{2}{2-p}} \quad \text { for all } 1 \leq p<2
$$

Remark 3.6. If we consider $p=1$ in Theorem 1.14, then we get the estimate obtained by Gröchenig (Ref. 10, Prop. 2.5.2). Thus, Theorem 1.14 generalizes the estimate of Gröchenig. In addition, if we compare Theorems 1.13 and 1.14, then we see that Theorem 1.14 gives a slightly sharper estimate.

\section{ACKNOWLEDGMENTS}

The author is deeply indebted to Professor S. Thangavelu for several fruitful discussions and generous comments. The author also wishes to thank Professor Aline Bonami for several valuable comments and suggestions concerning Theorems 1.11 and 1.12. Furthermore, the author is grateful to the University Grants Commission, India, for providing the Dr. D. S. Kothari Post Doctoral Fellowship [Award No. F.4-2/2006 (BSR)/MA/18-19/0032].

\section{AUTHOR DECLARATIONS}

\section{Conflict of Interest}

The author has no conflicts of interest to disclose. 


\section{DATA AVAILABILITY}

Data sharing is not applicable to this article as no new data were created or analyzed in this study.

\section{REFERENCES}

${ }^{1}$ L. Hörmander, “A uniqueness theorem of Beurling for Fourier transform pairs," Ark. Mat. 29, 237-240 (1991).

${ }^{2}$ S. Thangavelu, An Introduction to the Uncertainty Principle, Progress in Mathematics Vol. 217 (Birkhäuser, Basel, 2004).

${ }^{3}$ G. H. Hardy, "A theorem concerning Fourier transforms," J. London Math. Soc. s1-8, 227-231 (1933).

${ }^{4}$ M. Cowling and J. F. Price, "Generalizations of Heisenberg's inequality," in Harmonic Analysis, Lecture Notes in Mathematics Vol. 992, edited by G. Mauceri, F. Ricci, and G. Weiss (Springer, Berlin, 1983), pp. 443-449.

${ }^{5}$ A. Bonami, B. Demange, and P. Jaming, "Hermite functions and uncertainty principles for the Fourier and the windowed Fourier transforms," Rev. Mat. Iberoam. 19, 23-55 (2003).

${ }^{6}$ V. Havin and B. Jöricke, The Uncertainty Principle in Harmonic Analysis, A Series of Modern Surveys in Mathematics Vol. 28 (Springer-Verlag, Berlin, 1994).

${ }^{7}$ K. Gröchenig and G. Zimmermann, "Hardy's theorem and the short-time Fourier transform of Schwartz functions," J. London Math. Soc. 63(1), 205-214 (2001).

${ }^{8}$ B. Demange, "Uncertainty principles for the ambiguity function," J. London Math. Soc. 72(3), 717-730 (2005).

${ }^{9} \mathrm{~K}$. Gröchenig, Foundations of Time-Frequency Analysis (Birkhäuser, Boston, 2001).

${ }^{10} \mathrm{~K}$. Gröchenig, in Uncertainty Principles for Time-Frequency Representations, Advances in Gabor Analysis, edited by H. G. Feichtinger and T. Strohmer (Birkhäuser, Boston, 2003), pp. 11-30.

${ }^{11}$ D. L. Donoho and P. B. Stark, “Uncertainty principles and signal recovery,” SIAM J. Appl. Math. 49(3), 906-931 (1989).

${ }^{12}$ P. Jaming, "Principe d'incertitude qualitatif et reconstruction de phase pour la transformée de Wigner," C. R. Acad. Sci., Ser. I: Math. 327(3), 249-254 (1998).

${ }^{13}$ E. H. Lieb, "Integral bounds for radar ambiguity functions and Wigner distributions," J. Math. Phys. 31(3), 594-599 (1990).

${ }^{14}$ E. Malinnikova, "Orthonormal sequences in $L^{2}\left(\mathbb{R}^{d}\right)$ and time frequency localization," J. Fourier Anal. Appl. 16(6), 983-1006 (2010). 